\title{
Direct Observation of Icosahedral Clusters in Quasicrystals and Crystals
}

\author{
Eiji Abe $^{* * *}$ S. J. Pennycook ${ }^{*}$ and A. P. Tsai ${ }^{* *}$ \\ * Oak Ridge National Laboratory, Solid State Division, P.O. Box 2008, Oak Ridge, TN37831-6031 \\ USA \\ National Institute for Materials Science, Aperiodic Solids Research Team, 1-2-1, Sengen, \\ Tsukuba 305-0047, Japan
}

Quasicrystals are currently believed to be cluster-based structure; the noncrystallographic symmetry of the robust icosahedral atom clusters force their non-periodic arrangement, leading to formation of icosahedral quasicrystals. This picture is supported by the fact that some crystalline phases formed at close compositions of quasicrystals are in fact constructed by the periodic arrangement of large icosahedral atomic clusters.

Unfortunately, for the icosahedral quasicrystals, the image contrasts reflect the projected 3-dimensional quasiperiodic structure, so that we cannot derive the atomic model directly from the projected images [1]. However, a direct structural observation is still essential even for the icosahedral quasicrystals to converge a convincing atomic structure, because diffraction intensity method cannot give a unique solution (multi-solution problem due to a local isomorphic nature of a quasilattice constructed by a set of multiple unit cells). Here we demonstrate that, for the icosahedral $\mathrm{Zn}_{6} \mathrm{Mg}_{3} \mathrm{Ho}$ compound, the observed Z-contrast images are fairly well explained by the projected potential of only the Ho atomic arrangement [2], which was recently proposed by X-ray diffraction analysis; Ho occupies an even-body-center site of the 3-dimensional Penrose lattice [3].

Consequently, the present direct structural observation strongly supports the validity of the proposed Ho site. We note that Ho atoms do not form the multi-shell icosahedral clusters; multi-shell-like contrasts is a result of projection of the atomic configuration shown in FIG. 1.

Recently, a novel quasicrystalline compound has been discovered in binary $\mathrm{Cd}-\mathrm{Yb}$ and $\mathrm{Cd}-\mathrm{Ca}$ systems $[4,5]$. Interestingly, a cubic crystal composed of the icosahedral atomic cluster exists at very close composition. The atomic configuration in terms of multiple-shell inflation is not Mackay or Bergmann-type clusters, and thus the present cluster should be of a new type. Significantly, the atoms at the core $\left(1^{\text {st }}\right.$ shell) break the icosahedral symmetry (FIG.2). However, a cubic arrangement proposed by x-ray analysis is still controversial because the 4 atoms are actually located at the center due to 0.5 occupation probability for each cube site; a stable arrangement would be not partially-occupied cubic but a tetrahedron [4] or else as a local configuration. Here we directly observe the icosahedral cluster to see where and how atoms are arranged at the center. For observation, HAADF-STEM is a very good tool that provides incoherent images at atomic-resolution, a direct representation of the specimen scattering power without any artifacts. Preliminary observation suggests that $\mathrm{Cd}$ atoms do not form the cubic arrangement (FIG.2) [6]. We will discuss the favorable atomic configuration based on the EELS measurement and theoretical calculations.

\section{References}

[1] E. Abe and A. P. Tsai, Phys. Rev. Lett. 83 (1999) 753.

[2] E. Abe, H. Takakura and A. P. Tsai, J. Electron Microscopy, 50(3) (2001) 187.

[3] H. Takakura, M. Shiono, T. J. Sato, A. Yamamoto and A. P. Tsai, Phys. Rev. Lett. 86 (2000) 236.

[4] A. P. Tsai, J.Q. Guo, E. Abe, H. Takakura and T. J. Sato, Nature 408 (2000) 537.

[5] J. Q. Guo, E. Abe and A. P. Tsai, Phys. Rev. B 62 (2000) R14605.

[6] E. Abe, S. J. Pennycook and A. P. Tsai, unpublished 

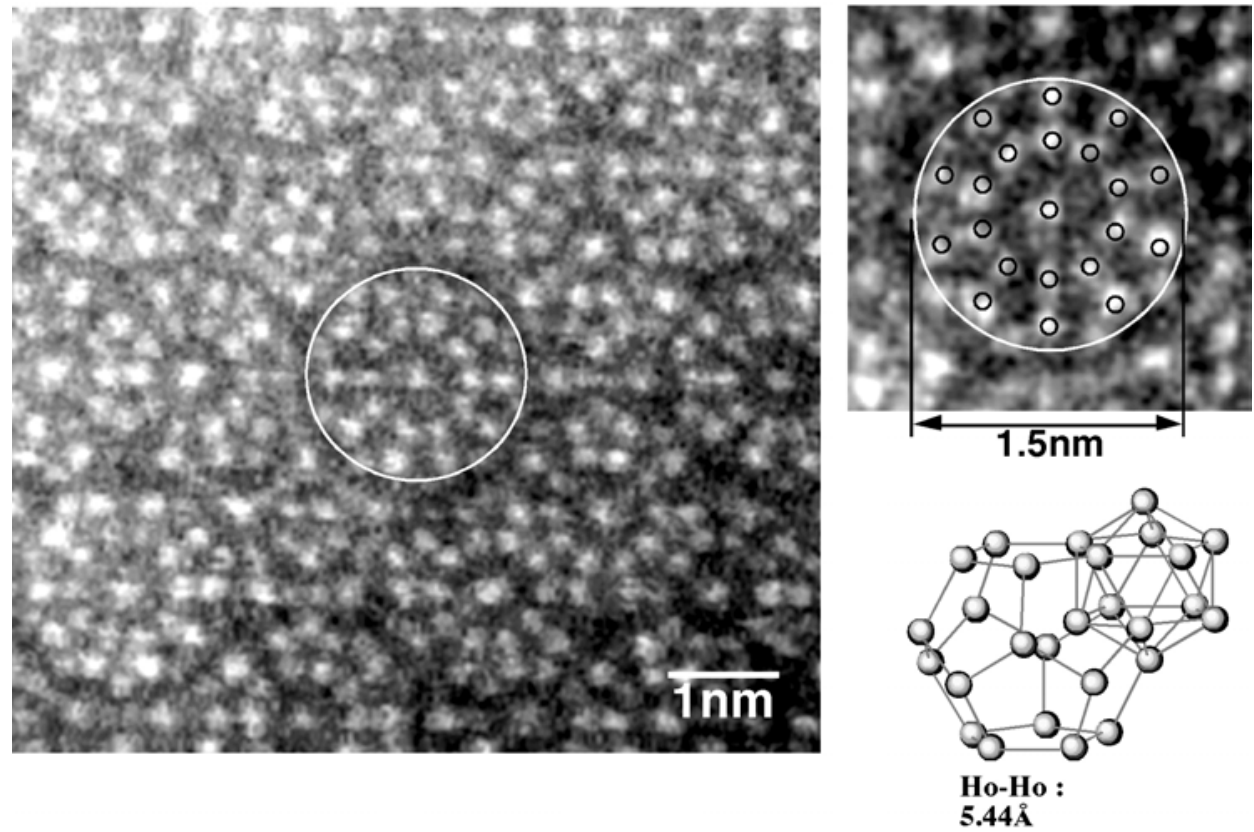

FIG. 1. Atomic-resolution Z-contrast (JEM-2010F with $\mathrm{Cs}=0.5 \mathrm{~mm})$ image taken along the fivefold symmetry axis of the $\mathrm{Zn}_{6} \mathrm{Mg}_{3} \mathrm{Ho}$ icosahedral quasicrystal [2]. A representative local Ho configuration explains well the observed tenfold symmetry Z-contrasts (encircled).

\section{Atomic cluster in $\mathrm{Cd}-\mathrm{Ca}$ cubic crystal}
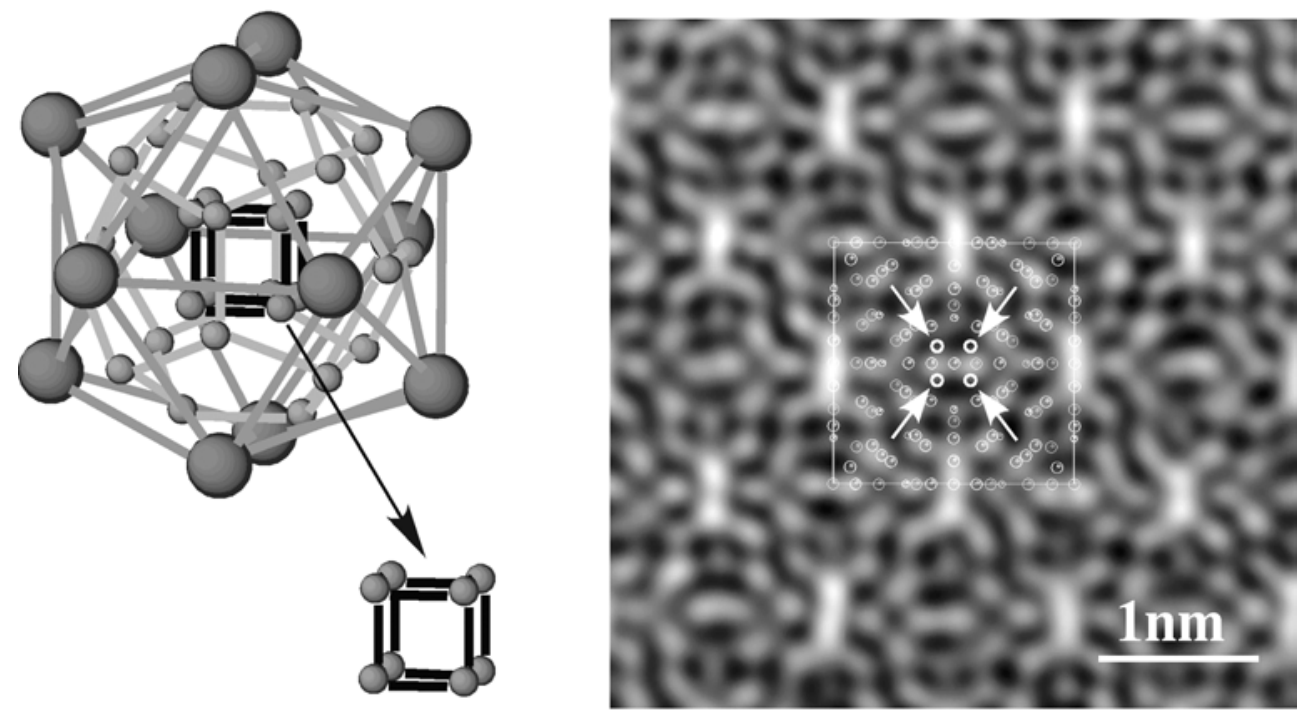

1st shell

FIG. 2. Icosahedral atomic cluster in the $\mathrm{Cd}_{6} \mathrm{Ca}$ cubic crystal and atomic-resolution Z-contrast (JEM-2010F with $\mathrm{Cs}=0.5 \mathrm{~mm})$ image taken along [100]. Note that no significant contrasts at the cubic sites (indicated by arrows), suggesting that $\mathrm{Cd}$ atoms are not located at these atomic sites. 